\title{
Quantum phase transition of the randomly diluted Heisenberg antiferromagnet on a square lattice
}

\section{$\operatorname{AUTHOR}(\mathrm{S}):$}

Kato, K.; Miyashita, S.; Todo, S.; Harada, K.; Kawashima, N.; Takayama, $\mathrm{H}$.

\section{CITATION:}

Kato, K....[et al]. Quantum phase transition of the randomly diluted Heisenberg antiferromagnet on a square lattice. Physical Review Letters 2000, 84(18): $4204-4207$

\section{ISSUE DATE:}

2000-05-01

URL:

http://hdl.handle.net/2433/200798

RIGHT:

(C) 2000 The American Physical Society 


\title{
Quantum Phase Transition of the Randomly Diluted Heisenberg Antiferromagnet on a Square Lattice
}

\author{
K. Kato,${ }^{1}{ }^{*}$ S. Todo, ${ }^{1}$ K. Harada,${ }^{2}$ N. Kawashima,${ }^{3}$ S. Miyashita, ${ }^{4}$ and H. Takayama ${ }^{1}$ \\ ${ }^{1}$ Institute for Solid State Physics, University of Tokyo, Kashiwa, 277-8581, Japan \\ ${ }^{2}$ Department of Applied Analysis and Complex Dynamical Systems, Kyoto University, Kyoto 606-8501, Japan \\ ${ }^{3}$ Department of Physics, Tokyo Metropolitan University, Tokyo 192-0397, Japan \\ ${ }^{4}$ Department of Applied Physics, University of Tokyo, Tokyo 113-8656, Japan
}

(Received 26 May 1999)

\begin{abstract}
Ground-state magnetic properties of the diluted Heisenberg antiferromagnet on a square lattice are investigated by means of the quantum Monte Carlo method with the continuous-time loop algorithm. It is found that the critical concentration of magnetic sites is independent of the spin size $S$, and equal to the two-dimensional percolation threshold. However, the existence of quantum fluctuations makes the critical exponents deviate from those of the classical percolation transition. Furthermore, we found that the transition is not universal, i.e., the critical exponents significantly depend on $S$.
\end{abstract}

PACS numbers: 75.10.Jm, 75.10.Nr, 75.40.Cx, 75.40.Mg

Since the discovery of high-temperature superconductivity in cuprates [1], effects of nonmagnetic impurities in two-dimensional Heisenberg antiferromagnets (HAF's) have been studied extensively. As is well known, the antiferromagnetic (AF) long-range order in pure $\mathrm{La}_{2} \mathrm{CuO}_{4}$ $(S=1 / 2 \mathrm{HAF})$ is immediately destroyed by a small amount of $\mathrm{Sr}$ substitution for La. Itinerant holes, doped into the $\mathrm{CuO}_{2}$ plane, cause magnetic frustration among $\mathrm{Cu} S=1 / 2$ spins and destroy the AF order. On the other hand, the AF long-range order is much robust [2-5] against static magnetic impurities such as $S=0 \mathrm{Mg}$ or $\mathrm{Zn}$ in $\mathrm{La}_{2} \mathrm{CuO}_{4}$, and $\mathrm{Mg}$ in $\mathrm{K}_{2} \mathrm{CoF}_{4}(S=1 / 2$ Ising AF) or $\mathrm{K}_{2} \mathrm{MnF}_{4}$ ( $S=5 / 2 \mathrm{HAF}$ ). However, it is notable that the critical concentration of nonmagnetic impurities strongly depends on the system in these cases. The critical concentration of $\mathrm{La}_{2} \mathrm{Cu}_{1-x} \mathrm{Mg}_{x} \mathrm{O}_{4}$ and $\mathrm{La}_{2} \mathrm{Cu}_{1-x} \mathrm{Zn}_{x} \mathrm{O}_{4}$ is observed [4] as $x \sim 20 \%$. It makes a sharp contrast with the others $[2,3]$, where the critical concentration is almost equal to the two-dimensional percolation threshold $(x \sim 40 \%)$. In $\mathrm{La}_{2} \mathrm{Cu}_{1-x} \mathrm{Mg}_{x} \mathrm{O}_{4}$ and $\mathrm{La}_{2} \mathrm{Cu}_{1-x} \mathrm{Zn}_{x} \mathrm{O}_{4}$, a quantum disordered phase may be realized due to strong quantum fluctuations.

These experimental results, and also purely theoretical interests, have motivated recent theoretical and also numerical studies [6-8] on the site-diluted HAF on the square lattice:

$$
\mathcal{H}=J \sum_{\langle i, j\rangle} \epsilon_{i} \epsilon_{j} \mathbf{S}_{i} \cdot \mathbf{S}_{j}
$$

Here, $\mathbf{S}_{i}=\left(S_{i}^{x}, S_{i}^{y}, S_{i}^{z}\right)$ denotes the quantum spin- $S$ operator at site $i$, and the nearest-neighbor coupling constant is antiferromagnetic $(J>0)$. The quenched dilution factors $\left\{\epsilon_{i}\right\}$ independently take 1 or 0 with probability $p$ and $1-p$, respectively, where $p(=1-x)$ denotes the concentration of magnetic sites.

In the classical case $(S=\infty)$, the present model at zero temperature is equivalent to the site-percolation prob- lem [9]. It is well known that the system undergoes a second-order phase transition at the percolation threshold $p_{\mathrm{cl}}$, which is determined as

$$
p_{\mathrm{cl}}=0.5927460(5)
$$

by the most recent simulation [10]. Near $p_{\mathrm{cl}}$, the staggered magnetization vanishes as $M_{\mathrm{s}} \sim\left(p-p_{\mathrm{cl}}\right)^{\beta_{\mathrm{cl}}}$ with $\beta_{\mathrm{cl}}=$ $5 / 36=0.13888 \ldots$ [9].

The main subjects in the previous works [6-8] have been focused on whether the critical concentration of the quantum systems $(S<\infty)$, referred to as $p^{*}$ hereafter, is identical to that of the classical model, $p_{\mathrm{cl}}$, or not, and also on its dependence on the strength of quantum fluctuations specified by the spin size $S$. For $S=1 / 2$, $p^{*}>p_{\mathrm{cl}}$ is suggested in these studies [6-8,11]. For example, $p^{*}=0.655$ and 0.695 are obtained in [6] and [8], respectively. However, they are still inconclusive and critical properties of the quantum phase transition and the quantum disordered phase (if exists) between $p^{*}$ and $p_{\mathrm{cl}}$ have not been well understood.

In this paper, we report results of large-scale quantum Monte Carlo (QMC) simulations on the diluted HAF (1) with $S=1 / 2,1,3 / 2$, and 2 . It is found, as we see below, that the AF long-range order at $T=0$ persists so long as a cluster of magnetic sites percolates, that is, $p^{*}=p_{\mathrm{cl}}$, even in the $S=1 / 2$ case. However, we find the critical exponents of the quantum phase transition just at $p_{\mathrm{cl}}$ are clearly different from those of the classical transition; the critical exponents vary depending on the value of $S$.

In the present QMC simulation, use of the continuoustime loop algorithm [12-15] is crucial. It greatly reduces correlations between successive world-line configurations, and therefore makes it possible to perform highly reliable simulations on large lattices (up to $L \times L=48 \times 48$ ) at extremely low temperatures $(T \sim 0.001 J)$. Another important feature of the present algorithm is its ergodicity; the winding number of world lines around vacant sites can 
change and the ground-canonical ensemble can be simulated. We use the periodic boundary conditions. For each sample, $10^{4}$ Monte Carlo steps (MCS) are spent for measurement after $10^{3}$ MCS for thermalization. At each parameter set $(L, T, p)$, physical quantities are averaged over 100-1000 samples.

First, we discuss the zero-temperature staggered magnetization $M_{\mathrm{s}}(p)$ for $p>p_{\mathrm{cl}}$, which is calculated as

$$
M_{\mathrm{S}}^{2}(p)=\lim _{L \rightarrow \infty} \lim _{T \rightarrow 0} \frac{3 S_{\mathrm{s}}(L, T, p)}{L^{d}}
$$

in terms of the static structure factor defined by

$$
S_{\mathrm{s}}(L, T, p)=\frac{1}{L^{d}} \sum_{i, j} e^{i \vec{k} \cdot\left(\vec{r}_{i}-\vec{r}_{j}\right)}\left\langle S_{i}^{z} S_{j}^{z}\right\rangle
$$

at the momentum $\vec{k}=(\pi, \pi)$, where $d$ is the spatial dimension $(d=2)$. The bracket in Eq. (4) denotes both the thermal average and the average over samples. In the present simulation, we use an improved estimator to calculate $S_{\mathrm{s}}(L, T, p)$, by which the variance of data is greatly reduced.

At temperatures lower than the gap of a finite system, $S_{\mathrm{s}}(L, T, p)$ converges to its zero-temperature value quite rapidly (probably exponentially). To obtain $S_{\mathrm{s}}(L, T=$ $0, p)$ at each $p$ and $L$, we perform QMC simulations at low enough temperatures so that $S_{\mathrm{s}}(L, T, p)$ exhibits no temperature dependence besides statistical errors. Note that as $p$ decreases the gap of a system of linear size $L$ becomes smaller, and therefore lower temperature is needed [16]. For the $S=1 / 2$ case, $T$ is taken as $0.002 J$ at $p=0.625$ and $L=48$.

In Fig. 1 , we plot $S_{\mathrm{s}}(L, 0, p) / L^{d}$ against $1 / L$ for $0.625 \leq p \leq 1$ in the $S=1 / 2$ case. It is clearly seen that the data at each concentration fall on a straight line for large $L$. In the clean system $(p=1)$, the leading finite-size correction is shown to be of $O(1 / L)$ according to the spin-wave theory [17]. The similar behavior for

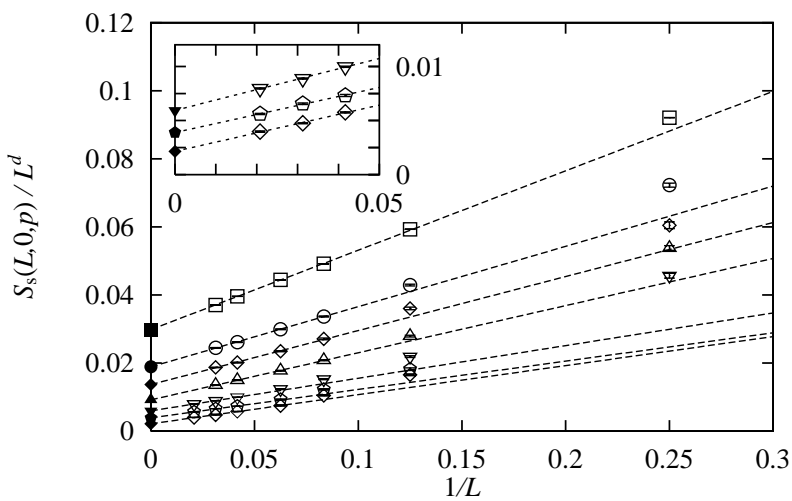

FIG. 1. System-size dependence of $S_{\mathrm{s}}(L, T=0, p) / L^{d}$ in the case of $S=1 / 2$ at $p=1,0.875,0.8125,0.75,0.6875,0.65$, and 0.625 (from upper to lower). The dashed lines are obtained by least-squares fitting for the largest three system sizes for each $p$. The extrapolated values are denoted by solid symbols. The data with $p \leq 0.6875$ and $L \geq 24$ are also shown in the inset. $p<1$ indicates that there exists an AF long-range order with massless excitations, such as spin waves, even in the presence of impurities. Thus, the staggered magnetization in the thermodynamic limit is obtained by linear extrapolation in $1 / L$ for the three largest system sizes at each $p$.

The final results for the zero-temperature staggered magnetization are shown in Fig. 2. It is seen that the staggered magnetization remains finite even at $p=0.625$ both in the $S=1 / 2$ and 1 cases. The possibility that $p^{*}$ is greater than 0.625 is excluded definitely, and the behavior of the whole magnetization curve strongly suggests that the critical concentration is identical to the percolation threshold $\left(p^{*}=p_{\mathrm{cl}}\right)$. Actually, the staggered magnetization seems to vanish algebraically towards $p=p_{\mathrm{cl}}$ as seen in the inset of Fig. 2. By least-squares fitting, we estimate the critical exponent $\beta$ as 0.46(3) and 0.32(3) for $S=1 / 2$ and 1 , respectively. Our conjecture, $p^{*}=p_{\mathrm{cl}}$, is supported more convincingly by the power-law behavior of the zero-temperature static structure factor just at $p=p_{\mathrm{cl}}$ :

$$
S_{\mathrm{s}}\left(L, 0, p_{\mathrm{cl}}\right) \sim L^{\Psi}
$$

with $\Psi=1.17(6)$ and 1.57(3) for $S=1 / 2$ and 1 , respectively. This is shown in Fig. 3, where no tendency of saturation of $S_{\mathrm{s}}\left(L, 0, p_{\mathrm{cl}}\right)$ is seen at all up to $L=48$.

The critical exponents $\beta$ and $\Psi$, obtained by the above analysis, differ from those of the percolation model $(\beta=$ $5 / 36$ and $\Psi=43 / 24$ [9]), that is, the universality class of the quantum phase transition at $p=p_{\mathrm{cl}}$ is different from the classical one. Furthermore, we found that the values of the critical exponents for $S=1 / 2,1$, and $\infty$ clearly differ from each other. This means that not only the fractal nature of the lattice geometry, but also the existence of quantum fluctuations and their strength, or the value of $S$, is relevant to the criticality.

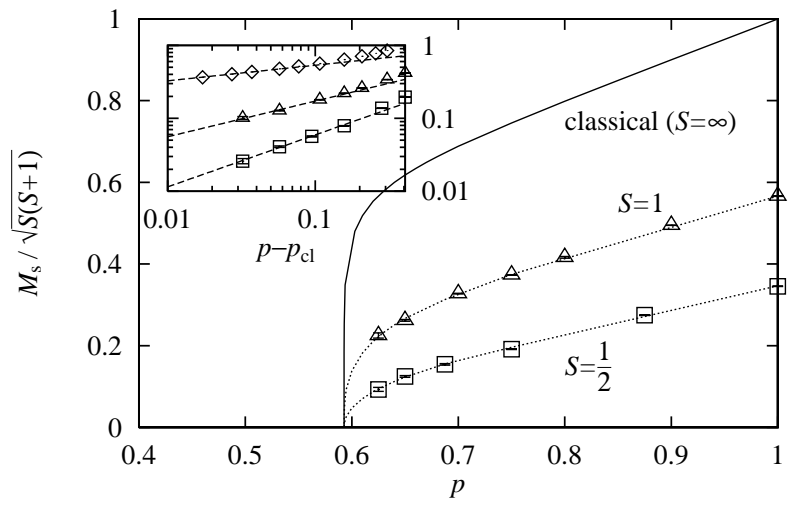

FIG. 2. Concentration dependence of the (normalized) staggered magnetization in the cases of $S=1 / 2$ (square) and 1 (triangle). The dotted lines are guides for the eye. In the inset, we show the double-logarithmic plot of the staggered magnetization against $\left(p-p_{\mathrm{cl}}\right)$. The dashed lines are obtained by least-squares fitting for $p \leq 0.70$. For comparison, the staggered magnetization in the classical limit is also plotted by the solid line and diamonds. 


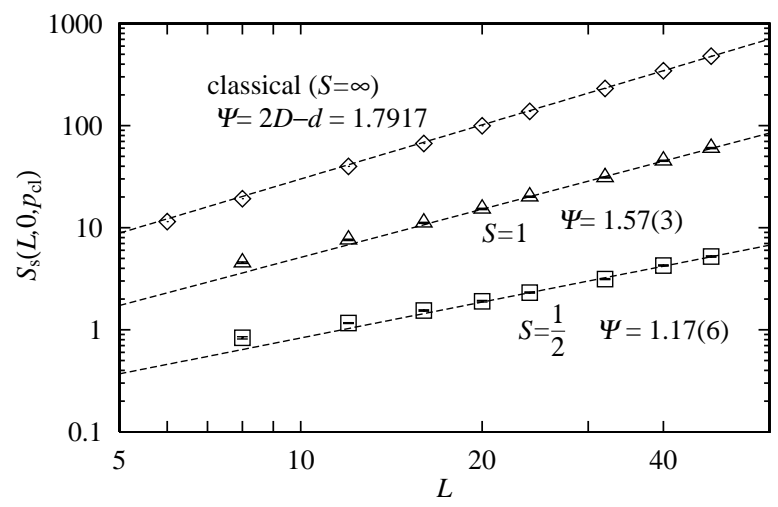

FIG. 3. System-size dependence of $S_{\mathrm{s}}(L, T=0, p)$ at $p=$ $p_{\mathrm{cl}}$ in the cases of $S=1 / 2$ (square) and 1 (triangle). The dashed lines are obtained by least-squares fitting for $L \geq 24$. The slope of the lines gives the exponent $\Psi$ [Eq. (5)]. The data in the classical limit are also plotted by diamonds.

The critical properties of the quantum phase transition found above may be described by the following scaling argument which is based on two assumptions on the quantum spin correlation on spin clusters. At $p=p_{\mathrm{cl}}$, all clusters are fractal with a fractal dimension $D$ (in two dimensions, $D=91 / 48$ ) [9]. First, we assume that the staggered spin correlation between two sites, $i$ and $j$, on a cluster behaves as

$$
C(i, j) \sim r_{i, j}^{-\alpha} \text { for } r_{i, j} \gg 1,
$$

where $r_{i, j}=\left|\vec{r}_{i}-\vec{r}_{j}\right|$. Here, we introduce a new $S$ dependent exponent $\alpha=\alpha(S) \geq 0$. In the classical case, $C(i, j)$ takes a constant independent of $r_{i, j}$, i.e., $\alpha(\infty)=0$. Together with the cluster-size distribution at $p=p_{\mathrm{cl}}$, predicted by the percolation theory [9], we obtain

$$
S_{\mathrm{s}}\left(L, 0, p_{\mathrm{cl}}\right)=\frac{1}{L^{d}} \sum_{\Omega} \sum_{i, j \in \Omega} C(i, j) \sim L^{2 D-d-\alpha},
$$

where the first summation is taken over clusters on the lattice. Comparing Eq. (7) with Eq. (5), we obtain a scaling relation:

$$
\Psi=2 D-d-\alpha .
$$

On the other hand, for $p>p_{\mathrm{cl}}$, the percolation theory [9] says that there exists a characteristic length $\lambda(p)$, below which the percolated cluster exhibits a fractal nature similar to that of clusters at $p=p_{\mathrm{cl}}$, but it has the ordinary dimension $(d=2)$ at the longer length scale. For $L \gg \lambda(p), S_{\mathrm{s}}(L, T, p)$ is dominated by the percolated cluster. We introduce the second assumption that there exist no other macroscopic characteristic lengths except $\lambda(p)$ even in the $S<\infty$ cases. Since there exists an AF long-range order as shown before, the correlation function is expected to obey the following scaling form [18]:

$$
\begin{aligned}
C(i, j ; p) & \sim r_{i, j}^{-\alpha} \tilde{C}\left[r_{i, j} / \lambda(p)\right] \\
& \sim \lambda(p)^{-\alpha} \text { for } r_{i, j} \gg \lambda(p),
\end{aligned}
$$

where $\tilde{C}(x)=$ const for $x \rightarrow 0$, so that Eq. (6) is reproduced for $1 \ll r_{i, j} \ll \lambda(p)$.

Using the fact that the characteristic length scale $\lambda(p)$ diverges as $\left(p-p_{\mathrm{cl}}\right)^{-\nu}$ near $p_{\mathrm{cl}}$ with $\nu=4 / 3$ [9], and assuming the ordinary algebraic temperature dependence, one finally reaches a full finite-size scaling (FSS) form of the structure factor:

$$
S_{\mathrm{s}}(L, T, p) \sim L^{2 D-d-\alpha} \tilde{S}_{\mathrm{s}}\left[L^{1 / \nu}\left(p-p_{\mathrm{cl}}\right), L^{z} T\right],
$$

where the $S$-dependent scaling function $\tilde{S}_{\mathrm{s}}(x, y)$ takes a constant at $(x, y)=(0,0)$, and $\tilde{S}_{\mathrm{s}}(x, 0) \sim x^{-(2 D-2 d-\alpha) \nu}$ for $x \gg 1$. In Eq. (10), we introduce the dynamical exponent $z$, which relates the energy scale with the length scale [19]. In the present case, $z>1$ is expected, since the Lorentz invariance is broken due to the existence of impurities. Although the algebraic $T$ dependence assumed in Eq. (10) is not guaranteed a priori, finite-temperature data of the staggered structure factor at $p=p_{\mathrm{cl}}$ are well scaled for $S=1 / 2,1,3 / 2$, and 2 , by using our FSS form (10) with the exponents $\Psi$ and $z$ listed in Table I, as shown in Fig. 4. The values of $\Psi$ are consistent with those obtained by the FSS at $T=0$ [Eq. (5) and Fig. 3]. Note that not only $\Psi$ but also $z$ depend on $S$.

The critical exponent $\beta$ is also expressed in terms of $\alpha$. By taking $L \rightarrow \infty$ after taking the limit of $T \rightarrow 0$ in Eq. (10), one obtains

$$
2 \beta=-(2 D-2 d-\alpha) \nu .
$$

The values of $\nu$ calculated from $\Psi$ and $\beta$ through Eqs. (8) and (11) are consistent with $\nu=4 / 3$ (Table I), which supports the scaling argument proposed above.

In summary, we have investigated the ground-state phase transition of the diluted HAF with $S=1 / 2,1,3 / 2$, and 2. Contrary to the previous works $[6-8,11]$, our present QMC study has shown that the critical concentration is equal to the classical percolation threshold even in the $S=1 / 2$ case. Concerning the relation to experiments, it becomes clear that the present model (1) is too much idealized to predict the magnetic properties observed in the real materials. Other effects, such as next-nearest-neighbor interaction, should be included properly into the model Hamiltonian. On the other hand, in the theoretical point

TABLE I. Summary of critical exponents $\Psi, z, \beta$, and $\nu$. $\Psi$ and $z$ are obtained by the FSS shown in Fig. 4 , and $\beta$ is estimated from the staggered magnetization (Fig. 2). The exponent $\nu$ is calculated from $\beta$ and $\Psi$ thorough the scaling relations, Eqs. (8) and (11).

\begin{tabular}{ccccc}
\hline \hline$S$ & $\Psi$ & $z$ & $\beta$ & $\nu$ \\
\hline $1 / 2$ & $1.27(2)$ & $2.54(8)$ & $0.46(3)$ & $1.2(1)$ \\
1 & $1.57(3)$ & $1.58(10)$ & $0.32(3)$ & $1.5(2)$ \\
$3 / 2$ & $1.60(3)$ & $1.55(10)$ & $\ldots$ & $\cdots$ \\
2 & $1.69(7)$ & $1.31(20)$ & $\ldots$ & $\cdots$ \\
$\infty$ & 1.79167 & $\ldots$ & 0.13889 & 1.33333 \\
\hline \hline
\end{tabular}



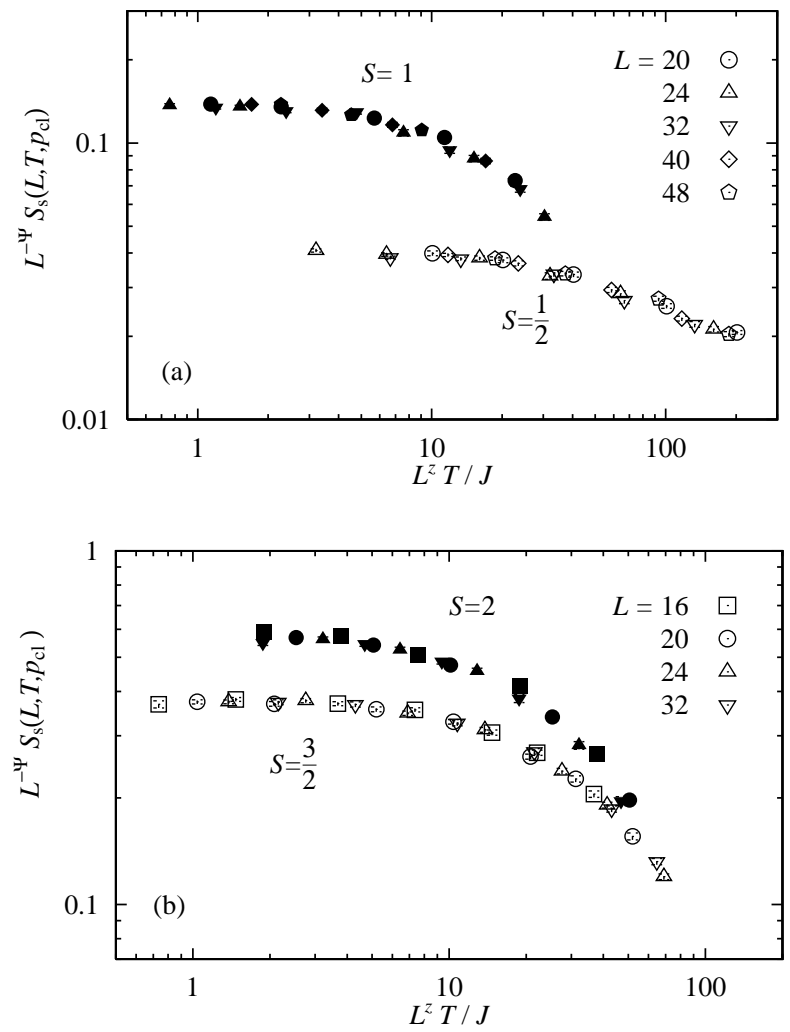

FIG. 4. Scaling plot of $S_{\mathrm{s}}(L, T, p)$ at $p=p_{\mathrm{cl}}$ for (a) $S=1 / 2$ and 1 , and for (b) $S=3 / 2$ and 2 .

of view, it has been revealed that the present model contains quite rich physics; the critical exponents vary depending on $S$. We have introduced a scaling form with two nonclassical exponents, $\alpha$ and $z$, which consistently explains the behavior of the simulation data together with the exponents $D$ and $\nu$ of the classical percolation transition. To our best knowledge, this is the first time that such an $S$-dependent quantum critical behavior is discovered.

Most of the numerical calculations for the present work have been performed on the CP-PACS at the University of Tsukuba, Hitachi SR-2201 at the Supercomputer Center, University of Tokyo, and on the RANDOM at the Materials Design and Characterization Laboratory, Institute for Solid State Physics, University of Tokyo. The present work is supported by the "Large-Scale Numerical Simulation Program" of the Center for Computational Physics, University of Tsukuba, and also by the "Research for the Future Program" (JSPS-RFTF97P01103) of the Japan Society for the Promotion of Science. N. K.'s work is sup- ported by a Grant-in-Aid for Scientific Research Program (No. 09740320) from the Ministry of Education, Science, Sport and Culture of Japan.

*Present address: Semiconductor Energy Laboratory Co., Ltd., Kanagawa, 243-0036, Japan.

[1] J. G. Bednorz and K. A. Mueller, Z. Phys. B 64, 189 (1986).

[2] L. J. de Jongh, in Magnetic Phase Transitions, edited by M. Ausloos and R. J. Elliott (Springer-Verlag, New York, 1983), p. 172.

[3] D. C. Johnston et al., Phys. Rev. B 36, 4007 (1987).

[4] S.-W. Cheong et al., Phys. Rev. B 44, 9739 (1991).

[5] M. Corti, A. Rigamonti, F. Tabak, P. Carretta, F. Licci, and L. Raffo, Phys. Rev. B 52, 4226 (1995).

[6] J. Behre and S. Miyashita, J. Phys. A 25, 4745 (1992); S. Miyashita, J. Behre, and S. Yamamoto, in Quantum Monte Carlo Methods in Condensed Matter Physics, edited by M. Suzuki (World Scientific, Singapore, 1994), p. 97.

[7] C. Yasuda and A. Oguchi, J. Phys. Soc. Jpn. 66, 2836 (1997); 68, 2773 (1999).

[8] Y.C. Chen and A. H. Castro Neto, Phys. Rev. B 61, R3772 (2000).

[9] D. Stauffer, Phys. Rep. 54, 1 (1979); D. Stauffer and A. Aharony, An Introduction to Percolation Theory (Taylor and Francis, London, 1985), 2nd ed., and references therein.

[10] R. M. Ziff, Phys. Rev. Lett. 69, 2670 (1992).

[11] For a bond-diluted HAF, $p^{*}>p_{\mathrm{cl}}$ is suggested again by A. W. Sandvik and M. Vekicać, Phys. Rev. Lett. 74, 1226 (1995).

[12] H. G. Evertz, in "Numerical Methods for Lattice Quantum Many-Body Problems," Frontier in Physics, edited by D. J. Scalapino (Perseus, Reading, to be published), and references therein.

[13] H. G. Evertz, G. Lana, and M. Marcu, Phys. Rev. Lett. 70, 875 (1993); U.-J. Wiese and H.-P. Ying, Z. Phys. B 93, 147 (1994); B. B. Beard and U.-J. Wiese, Phys. Rev. Lett. 77, 5130 (1996); N. Kawashima and G. E. Gubernatis, Phys. Rev. Lett. 73, 1295 (1994).

[14] K. Harada, M. Troyer, and N. Kawashima, J. Phys. Soc. Jpn. 67, 1130 (1998).

[15] S. Todo and K. Kato, e-print cond-mat/9911047.

[16] The temperatures $(T \sim 0.1 J)$ used in the previous QMC studies $[6,11]$ are too high to estimate correct zerotemperature staggered magnetization for small $p$.

[17] D. A. Huse, Phys. Rev. B 37, 2380 (1988).

[18] We omitted corrections from massless excitations in Eq. (9) for simplicity.

[19] J. A. Hertz, Phys. Rev. B 14, 1165 (1976). 